\title{
On The Modeling of Chemical Flow in Unsaturated Mobile-Immobile Water Systems
}

\author{
Uichiro Matsubayashi ${ }^{1}$, German T. Velasquez ${ }^{2}$, Fusetsu Takagi ${ }^{3}$
}

\begin{abstract}
In dealing with groundwater contamination and pollution monitoring, it is important to predict the characteristics of the various chemicals that flow in the soil-water environment. This research reviews the current state of the formulations of chemical flow in unsaturated mobile-immobile water systems and proposes a new approach towards the selection of a model basis. This includes the use of the irreducible moisture as the immobile moisture term and the use of a simple kinetic term for the soil sorption. These assumptions when tested on a simple discrete experiment for the sorption term and analytical and experimental fitting for the immobile term show an acceptable level of performance.
\end{abstract}

Keywords: Mobile-immobile model, chemical flow, unsaturated flow model.

\section{Introduction}

\subsection{General}

Solute movement studies are important tools in the field of subsurface hydrology especially in predicting the movement of pesticides, nitrates, heavy metals, leached salts from the surface layers of the soil and other solutes through the soil. Studies of this type provide information not only on the characteristics of the chemical being transported (dispersion, diffusion, sorption reactions, or other exchange processes) but also information about the medium itself (van Genuchten and Wierenga, 1976). By observing the characteristics of the function of the concentration at the outflow of experimental columns (breakthrough curves or BTCs), chemical characteristics have been determined (van Genuchten and Wierenga, 1976) and certain soil parameters were obtained (Sugita and Gillham, 1993). Concerning the formulation of the flow of water and chemicals in porous media, Biggar and Nielsen (1967), and Bear (1972) provides good reviews of the state of the art.

Most of the formulations, however, are based on the equations of continuity of water flow and the convective-dispersive equation of mass balance (Lapidus and Amundson, 1952). The equation of mass balance can be expressed as equation (1), for the general unsteady water flow, and equation (2) for steady state uniform flow $(\partial \theta / \partial z=0)$. In the above equation $C$ is the concentration of the flow $(\mathrm{g} /$ $\left.\mathrm{cm}^{3}\right), D_{d}$ is the dispersion coefficient $\left(\mathrm{cm}^{2} /\right.$ day), $q$ is the Darcy flux ( $\mathrm{cm} /$ day) and $v_{0}$ is the pore water velocity (Darcy flux divided by the volumetric water content $\theta ; \mathrm{cm} /$ day). Also, $z$ is the distance of flow $(\mathrm{cm})$ and $t$ is the time (days).

The use of equation (2) however, produces symmetrical concentration distributions both with depth and as BTCs (Gershon and Nir, 1969). Experiments on the other hand have consistently shown that concentration distributions are generally non-symmetrical or nonsigmoid in character (Aylore and Karim 1970, Green et al. 1972). This phenomenon is usually called tailing, indicating the appearance of such unsymmetrical concentration distributions (van Genuchten et al. 1976). Experiments have shown that the tailing phenomenon to occur under the following conditions; a) during unsaturated flow where larger pores become empty and thus contribute less to the total flow, b) during flow in aggregated media where there is usually slow and incomplete mixing between waters, c) during flows with low water velocity, and d) if sorption reactions exist (Nielsen and Biggar 1961, Biggar and Nielsen 1962, Giddings 1963, Gupta et al. 1973, MacMahon and Thomas 1974, Skopp and Warrick, 1974).

\subsection{Previous Formulations}

From the above discussions it is clear that equation (2) is incapable of predicting the actual process of unsaturated-saturated water and chemical flow in porous media. In order to account for the tailing phenomenon, several researchers have made modifications of equation (2) to include mobile and immobile water or dynamic and stagnant water zones with transfer or diffusion between the two zones (Deans 1963, Skopp and Warrick 1974). These formulations take different forms and considers differ-

\footnotetext{
' Associate Prof., Nagoya University, Chikusa-ku, Nagoya

${ }^{2}$ UN Research Fellow, United Nations Centre for Regional Devt., NIC, Nagoya Japan

${ }^{3}$ Professor, Nagoya University, Chikusa-ku, Nagoya
}

$\frac{\partial \theta C}{\partial t}=\frac{\partial}{\partial z}\left(D_{d} \theta \frac{\partial C}{\partial z}\right)-q \frac{\partial C}{\partial z}$
$\frac{\partial C}{\partial t}=D_{d} \frac{\partial^{2} C}{\partial z^{2}}-v_{o} \frac{\partial C}{\partial z}$
$\frac{\partial S}{\partial t}=\alpha(K C-S)$


ent parameters. Some of the most notable formulations are as follows; a) One formulation is from Coats and Smith (1964) who expanded the model by Deans (1963). In their formulation two soil-water regions are considered namely, the mobile and the immobile zones. The immobile part of the soil water is assumed to be created from the existence of dead end pores and trapped air inside the soil; b) Another formulation is from van Genuchten and Wierenga (1976) and De Smedt and Wierenga (1979) who proposed a general transport equation which includes reaction terms between the soil and mobile and immobile water. In the formulation, they assumed that the adsorption is instantaneous and that the relation between the solution concentration and adsorption can be described by a linear Freundlich relationship $(S=K C)$ which when introduced into equation (2) produces the retardation factors described by Hashimoto et al. (1964); c) The above formulations were further expanded by van Genuchten and Wagenet (1989), and Zurmuhl et al. (1991) to include solute decay composed of degradation terms for both the water and the soil, the functions of which are dependent on the type of chemical being modelled; d) Aside from these, there were other approaches, including a two phase model (Skopp and Warrick 1974), a classification of the water system into stagnant, moderately mobile and rapidly mobile waters (Horiuchi et al. 1992), and a dual porosity model (Gerke and van Genuchten 1993) to name a few. Solutions to some of these equations can be found in Coats and Smith (1964), Villermaux and Swaay (1969), Bennet and Goodridge (1970), van Genuchten and Wierenga (1976), Goltz and Roberts (1986), Lassey (1988), Toride et al. (1993), and Fry et al. (1993).

Although these kinds of relationships have been successfully used in modelling, most researchers admit that the selection of the model assumptions and the determination of the numerous parameters becomes difficult. In horizontal soil-column experiments by Smiles and Philip (1978), they have found that there seems to be a piston-like displacement of the initial water by the absorbed water, suggesting that there is very little or no immobile water. This means that for experimental soils there seems to be no basis for a separation of the system into mobile and immobile waters.

Even if the water can be initially assumed to be separated into mobile and immobile zones, as in trapped water in dead-ended pores as the immobile part, the determination of the mobile and immobile fractions would be very difficult especially during unsteady state flows. Another is the assumption that the sorption reactions are instantaneous. This would present problems for variable water velocities especially during transient states of the soil. If the rate of adsorption is slow compared to the rate by which the chemicals move through the soil, then a kinetic approach towards equilibrium would be more appealing. One formulation was proposed by Lapidus and Amundson (1952) as shown in equation (3). There are other formulations for the storage component of the flow equations comprising sorptionequilibrium, non-equilibrium and additive combinations of the two. A thorough review of formulations is given by Boast (1973), and of the underlying assumptions by van Genuchten and Wierenga (1976), Nkedi-Kizza et al. (1983), and Goltz and Roberts (1986).

\section{Basis of the Proposed Model}

To overcome some of the limitations of the above formulations, a new model is required that will utilize some physical basis thus minimizing the number of parameters which needs to be estimated. Based on this, a new model is proposed which rationalizes the selection of the immobile water component and the soil sorption term.

2.1 Formulation of Immobile Term

Considering the physics of the $\psi-\theta$ relationship, there would naturally exist an irreducible moisture content $\theta_{r}$ even during dry conditions whose value would vary depending on the soil type. For most soils, this amount is small as may be the case for certain bead-column experiments, but for some materials this moisture content is considerable and therefore cannot be neglected. Based on this, a new model is proposed utilizing the irreducible moisture content as the immobile component of the water system. Not only will this assumption provide the model with another physical basis, it will also provide an accurate value for the immobile moisture parameter, $\theta_{i m}$.

The use of this assumption would not contradict the results of other researchers who have found that there was very little or no immobile fraction, since the irreducible moisture for such experimental sands and beads are extremely small.

2.2. Checking Kinetic Sorption Approach by Experimental Fitting

It has been generally stated that physically, ions in aggregated soils may be viewed as soil salts in solution existing in pores between and inside the aggregates. Biggar and Nielsen (1967) stated that of the two kinds of pores, the larger in-between-the-soil pores dominate ion flow and mixing, while the in- 
Model 1: $\frac{d V S^{\prime}}{d t}=-\beta_{s m}\left(S^{\prime}-C\right)$
Model 2: $\frac{d V S^{\prime}}{d t}=-\beta_{s m}\left(S^{\prime}-C\right)^{n}$
Model 3: $\frac{d V S^{\prime}}{d t}=-\beta_{s m}\left(S^{\prime}-C\right)^{2}-\beta_{s m}^{\prime}\left(S^{\prime}-C\right)$

aggregate pores act as a kind of ion source or sink. Considering that the effect of immobile water is negligible for typical soils compared to soil sorption reactions, three discrete models are considered and compared with experimental results. The objective of this is to determine the feasibility of different formulations of a kinetic approach towards equilibrium.

The experiments were conducted on $40 \mathrm{~cm}$ long soil columns with rainwater as the initial input on top of the column and observation of the outflows at the bottom of the column. The experiments mimic the different states of discrete lengths of the soil during rainfall and observes the outflow concentration for two states. One is an Eulerian observation of the outflow from a soil length during repeated washing of water; and the other is a Lagrangian observation of the outflow as a unit volume of water flows along the soil slope. Details of the experimental setup can be found in Velasquez $(1991,1994)$ and Matsubayashi et al. (1992). The schematic diagram of the model system and the observations can be seen in figure 1. In the above, an Eulerian observation simply means that the observer is fixed at a point while the process flows along his view. In the Lagrangian observation, the observer flows along with the process. These two viewpoints are needed since the actual chemical-soil sorption process may either be that the soil has more concentration than the diluting water (similar to an Eulerian observation) or it may have less concentration than the diluting water (similar to a Lagrangian observation).

Three models are used to fit the experimental data, all of which are of a kinetic approach towards equilibrium. This was done to determine the performance of this compared to an equilibrium approach (e.g., linear Freundlich Isotherm) and to compare the performance of similar models of varying complexities. The first model used in the fitting is similar to that of Macpherson and Peck (1987) and considers that the amount of ions carried away in constant flow during a time increment $d t$ is proportional to the difference in concentration between the ion storage and the diluting flow. This model is given by equation (4). The second model is a non-linear modification of the first and is given by equation (5). The third model is a further modification where it is a non-linear series function as shown in equation (6). In these equations, $V$ is a representative volume (liters) for the ion stored in the soil and is assumed to be constant. $S$ ' is the virtual concentration of the in-aggregate pore water (g/liter), $C$ and $C 1$ are the concentration of the diluting flow and the outflow, respectively ( $\mathrm{g} /$ liter), $Q$ is the flow rate (liters/min), and $\alpha$ is a kinetic parameter (liter/min).

The results of the fitting is shown in figure 2 and Table 1 . As can be seen in Table 1, there is not much difference between the three models
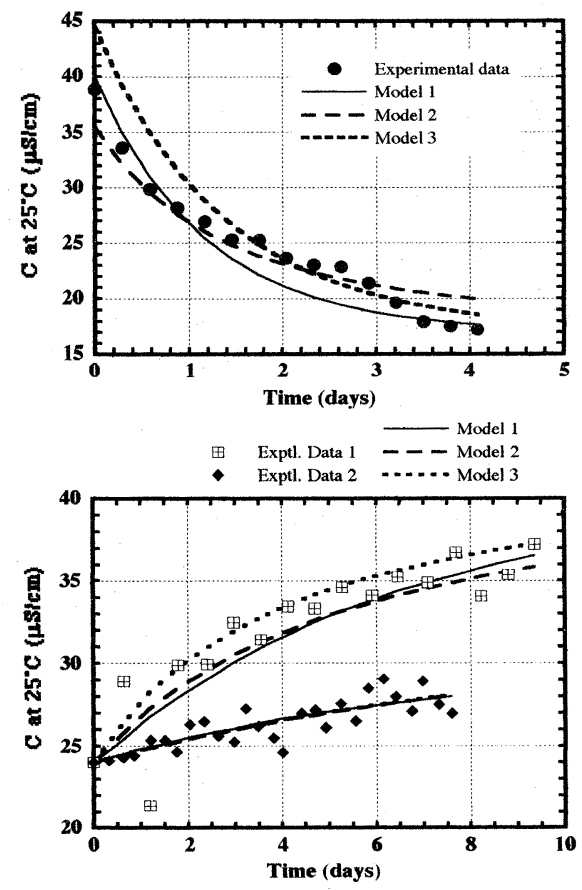

Fig. 2. Fitting of Models 1, 2 and 3 to the experimental Eulerian (top) and Lagrangian (bottom) observation data.
Table 1. Goodness of fit and sorption parameter values for models.

\begin{tabular}{|c|c|c|c|c|c|c|c|c|c|}
\hline \multirow{3}{*}{ Observation } & \multirow{3}{*}{ Model } & \multirow{2}{*}{\multicolumn{2}{|c|}{$\begin{array}{l}\text { Regression } \\
\text { Coefficient }\end{array}$}} & \multicolumn{6}{|c|}{ Parameters } \\
\hline & & & & \multicolumn{2}{|c|}{$\beta_{\mathrm{sm}}(\mathrm{I} / \mathrm{min})$} & \multicolumn{2}{|c|}{$\beta_{\mathrm{sm}}(\mathrm{L} / \mathrm{min})$} & \multicolumn{2}{|c|}{$\mathbf{n}(-)$} \\
\hline & & Data 1 & Data 2 & Data 1 & Data 2 & Data 1 & Data 2 & Data 1 & Data 2 \\
\hline \multirow{3}{*}{$\begin{array}{r}\text { Eulerian } \\
\text { Observation }\end{array}$} & 1 & 0.97 & - & 0.5 & - & - & - & - & - \\
\hline & 2 & 0.98 & - & 0.005 & - & - & - & 4 & - \\
\hline & 3 & 0.97 & - & 0.0005 & - & 0.4 & - & - & - \\
\hline \multirow{3}{*}{$\begin{array}{l}\text { Lagrangian } \\
\text { Observation }\end{array}$} & 1 & 0.89 & 0.83 & 0.035 & 0.0175 & - & - & - & - \\
\hline & 2 & 0.89 & 0.83 & 0.0015 & 0.0005 & - & - & 4 & 4 \\
\hline & 3 & 0.89 & 0.83 & 0.002 & 0.0005 & 0.005 & 0.005 & - & - \\
\hline
\end{tabular}


as far as goodness of fit is concerned. For the sorption kinetic parameters, it seems to be in the order of $10^{-2}$ or smaller for both the linear and non-linear cases.

\section{A New Model}

\subsection{Formulation}

Considering the above, for the flow of chemicals through an unsaturated, aggregated, sorbing porous media, four regions can be identified as shown in figure 3 . The regions are as follows: a) Air spaces trapped between soil particles; b) Mobile water located inside pore spaces both large and small. Solute flux occurs through advection and diffusion in this region; c) Immobile water which envelopes the soil particles. This enveloping water is the irreducible water film as described by $\theta_{r}$ in the $\psi-\theta$ relationship. This region mixes with the mobile region through a kinetic mixing term; d)

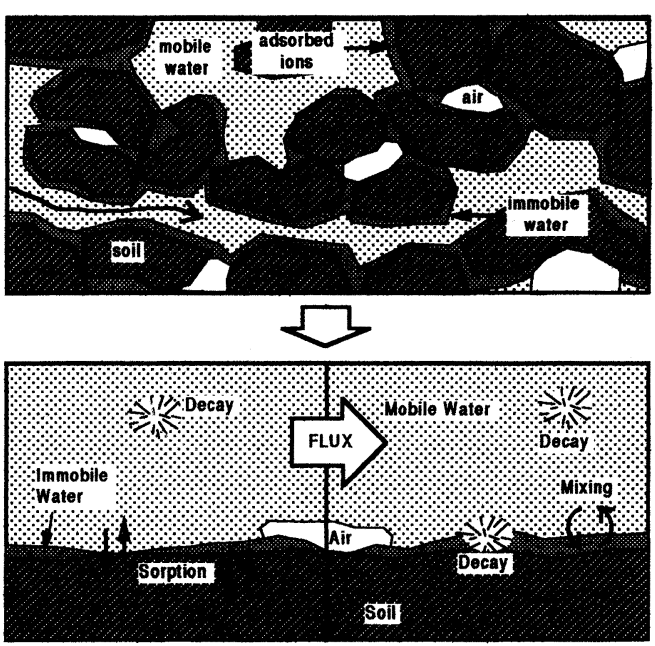

Fig. 3. Schematic diagram of new model. Soil region which is in contact with the immobile region and in some places with the mobile region. The percentage of contact of this region with the mobile part is given by the parameter $f$. Soil sorption reactions occur in this region with the immobile and mobile regions. Decay exists in the chemicals in the last three regions described by a first order decay term (for ions, decay may mean reaction with other ions forming signless-chemical molecules thus neutralizing them). For the relationship between the adsorbed and solution concentration, a kinetic approach to equilibrium is proposed.

The conservation equations thus becomes equation (7) for the mobile region, and equation (8) for the immobile region, with the relationship between $S$ and $C$ expressed in non-equilibrium form as in equation (9), where $C$ is the solution concentration $\left(\mathrm{g} / \mathrm{cm}^{3}\right), S$ is the adsoption in the soil region $(\mathrm{g} / \mathrm{g})$, $\alpha$ is a kinetic mass transfer coefficient $\left(\right.$ day $\left.^{1}\right), \rho$ is the soil bulk density $\left(\mathrm{g} / \mathrm{cm}^{3}\right)$ and $K$ is a dimensionadjusting coefficient $\left(\mathrm{cm}^{3} / \mathrm{g}\right)$. Thus for the total soil it becomes equation (10), where linear decay is assumed as equation (11). In these equations, $d_{m}, d_{i m}$ and $d_{s}$ are first order decay term for the mobile water, immobile water and soil, respectively $\left(\mathrm{g} / \mathrm{cm}^{3} /\right.$ day), $\beta_{s m}$, and $\beta_{s i}$ are kinetic sorption parameters between the soil and the mobile and immobile water $\left(\right.$ day $\left.^{-1}\right)$ and $\xi_{m}, \xi_{i m}$ and $\xi_{s}$ are decay parameters $\left(\right.$ day $\left.^{-1}\right)$. Also, $J\left(J=\theta_{m} C v-\theta D_{d} \operatorname{grad}(C)\right)$ is the solute flux by advection and dispersion $\left(\mathrm{g} / \mathrm{cm}^{2} /\right.$ day $)$.

3.2. Fitting the Model with Analytical and Experimental Data

The differential equations were solved using an alternative predictor-corrector finite difference scheme which retains all the advantages of a Crank-Nicolson approach but slightly increases the truncation error to $\mathbf{O}\left((\Delta \mathrm{x})^{2}+(\Delta \mathrm{t})^{3 / 2}\right)$ (Remson et al. 1971, Freeze 1978). To determine the performance of the model compared to experimental and analytical data, the model results were fitted with the analytical results from van Genuchten and Wierenga (1976) and experimental results from Shimojima and Sharma (1993).

The fitting with the analytical results can be seen in figure 4 . The analytical results were for the mobile-immobile model by van Genuchten and Wierenga (1976), where the soil-water sorption was estimated using the Freundlich isotherm. From figure 4 it can be seen that for almost the same model parameters, the numerical and analytical results vary by a lag of about 0.2 pore volumes. This can be explained by the fact that the Freundlich isotherm produces in-

$$
\begin{aligned}
& \frac{\partial \theta_{m} C_{m}}{\partial t}+f\left\{\frac{\partial \rho S}{\partial t}\right\}_{m}+\frac{\partial J}{\partial z}+\alpha\left(C_{m}-C_{i m}\right)-d_{m}=0 \\
& \frac{\partial \theta_{i m} C_{i m}}{\partial t}+(1-f)\left\{\frac{\partial \rho S}{\partial t}\right\}_{i m}-\alpha\left(C_{m}-C_{i m}\right)-d_{i m}=0 \\
& \left\{\frac{\partial \rho S}{\partial t}\right\}_{m}=\rho \beta_{s m}\left(K C_{m}-S\right) \text { and }\left\{\frac{\partial \rho S}{\partial t}\right\}_{i m}=\rho \beta_{s i}\left(K C_{i m}-S\right) \\
& \frac{\partial \rho S}{\partial t}=f \rho \beta_{s m}\left(K C_{m}-S\right)+(1-f) \rho \beta_{s i}\left(K C_{i m}-S\right)-d_{s} \\
& \left(d_{m}=\xi_{m} \theta_{m} C_{m}, d_{i m}=\xi_{i m} \theta_{i m} C_{i m}, d_{s}=\xi_{s} \rho S\right)
\end{aligned}
$$


stantaneous sorption reactions between the soil and the water causing a faster distribution of chemicals between the water and the soil thus the delay of the concentration breakthrough. However, besides the lag, it can be seen that the two curves are similar in shape.

For the experimental data fitting, the curves can be seen in figure 5 . The experimental results were for Bassendean sand columns with $11 \mathrm{ppm} \mathrm{PO}_{4}$-P used as the input solution. The soil properties are described in Taniguchi and Sharma (1990) and details of the experiments can be found in Shimojima and Sharma (1993). From figure 5, it can be seen that the numerical calculations predict well the experimental data even though it may seem to disagree with the data at the later stage of the experimental BTC. The disagreement of the prediction with the experiment at the later stage of the BTC may be entirely dependent on an unknown
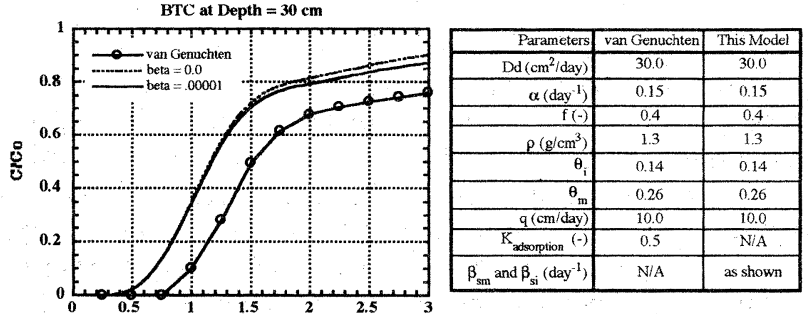
Pore Volume

Fig. 4. Fitting of numerical results with analytical solution by van Genuchten and Wierenga (1976).

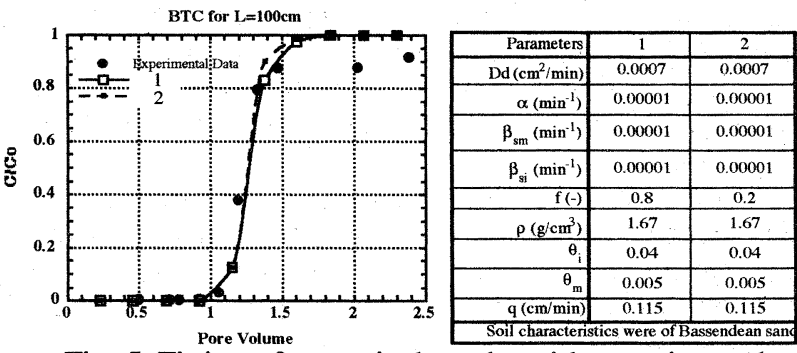

Fig. 5. Fitting of numerical results with experimental data of Shimojima and Sharma (1993). chemical process. Since it is physically unusual for an initially steep BTC to suddenly flatten below the input concentration under regular process of advection (the steep BTC means low dispersion) with no detectable sorption processes during initial breakthrough up to about 90 percent of the total input concentration, then the sudden flattening of the concentration at about 1.5 pore volumes cannot be explained by the present equations without further and more detailed knowledge of the experiment itself. However aside from the latter stage of the BTC, it can be seen that estimation of the immobile term using the irreducible moisture produces the desired lag of 0.25 pore volumes from the value 1.0 which should be the BTC's mid-position expected by the use of the usual mobile-only advection-dispersion equation of flow (since the parameters $\rho, \theta_{\mathrm{i}}, \theta_{\mathrm{m}}$, and $q$ are actual experimental values, and the rest of the parameters are practically very small). The result of the fitting shows that the fraction of the soil exposed to the mobile water zone does not greatly affect the BTC, mainly due to the very low sorption terms required for the estimated fitting. The dispersion coefficient was also very low which can be explained by the very steep BTC of the experiment.

\section{Discussions and Conclusions}

In an attempt to mimic the physical phenomenon of tailing, modifications have been made on the mass transport equation to include the effects of mobile and immobile waters and soil sorption reactions. However, in this attempt two points becomes prominent. These are whether to do away with such a mobile-immobile distinction of the water system and retain simple sorption reactions to simplify the modelling process, or whether to include both sorption and the mobile-immobile distinction and deal with the increased number of parameters through some kind of assumptions. It is obvious that the usual mass transport equations in itself cannot fully approximate the actual phenomenon and thus needs some modification, but to what extent this modification should be made still, up to the present, has not been fully resolved. It is obvious that for certain soils and for certain ions, one phenomenon may be more prominent than the other, but since this is not the case for all soils, thus there is a need for a more specific, non-general approach to these model basis formulation. Even for specific approaches in the determination of model parameters through experiments, unsuitable assumptions may cause erroneous results. In one example, the immobile component derived from the difference of the observed total moisture (through suction measurements by porous cup) and the mobile moisture (through the measurement of the time of arrival of effluent at a certain point in the column) may totally depend on the accuracy of the derived $\psi-\theta$ relationship of the soil (and the determination of the actual state of the experiment in the graph between the drying and wetting curves). As for the soil sorption term, since this is highly soil type dependent, a single generalization may not be suitable for all types of experimental and field usage. 
From these discussions it becomes clear that a simple physical basis is needed and will be applicable on a case to case basis. This research proposes such a basis from the results of the above analysis. One such proposal is the utilization of the irreducible moisture content for the immobile component of the water system. This will simplify its determination and its use will still be in agreement with the results of previous researchers. The second proposal is the use of a more simple formulation for a kinetic approach of the soil sorption term if the kinetic approach becomes suitable for the particular ion being studied. This is because of the results of the fitting to experimental results of three models of increasing complexity, all of which producing similar goodness of fitting.

What is still required is an experimental derivation of the applicability of the different formulations (equilibrium, non-equilibrium, addition of both) of the soil sorption reactions depending on different conditions. However, a clear understanding of the basis for such formulations can be used as an initial guide in modelling, as in the applicability of the linear and non-linear Freundlich isotherm, linear and non-linear kinetic approach, and the interaction of two or more ions. Nevertheless, since the more complex the sorption term becomes, the more the number of parameters have to be estimated or derived, then a determination of the balance between simplicity and formulation logic becomes highly important.

One approach which is now being undertaken by the authors is the formulation of the model through the above assumptions and then the derivation of the rest of the parameters through idealized experiments where the effect of other parameters are negligible or are nonexistent. It is hoped that through this kind of approach, the parameters of chemical flow in unsaturated soil can be more seriously analyzed.

\section{References}

[1] Aylmore, J.A.G. and M. Karim, Leaching ... columns. Int.Cong. of Soil Sci., Trans. 9th (Adelide Aust.) I, 143-153, 1970.

[2] Bear, J., Dynamics of fluids in porous media. American Elsevier, New York, 39-112, 1972.

[3] Bennet, A. and F. Goodridge, Hydrodynamic ... dispersion. Trans. of the Inst. of Chemical Engineering, 48, 232-244, 1970.

[4] Biggar, J.W. and D.R. Nielsen, Miscible ... of tracers. Soil Science Society American Proceedings. 26, 125-128, 1962.

[5] Biggar, J.W. and D. Nielsen, Miscible ... phenomena. In: Irri. of agri. lands. Agro., II, 254-274, Am. Soc. of Agr., Mad., Wis., 1967.

[6] Boast, C.W., Modelling the movement of chemicals in soil by water. Journal of Soil Science, 115, 224-230, 1973.

[7] Coats, K.H. and B.D. Smith, Dead-end pore ... in porous media. Society of Petroleum Engineering Journal, 4, 73-84, 1964.

[8] De Smedt, F. and P.J. Wierenga, Mass transfer in ... with immobile water. Journal of Hydrology, 41, 59-67, 1979.

[9] Deans, H.H., A mathematical model ... in porous media. Society of Petroleum Engineering Journal, 3, 49-52, 1963.

[10] Freeze, R.A., Mathematical ... hydrology. In: M.J. Kirkby (Editor), Hillslope Hydrology, Wiley-Intersci., 182-194, 1978.

[11] Gerke, H. and M. van Genuchten, A Dual-Porosity ... Porous Media. Water Res. Research, 29, 305-319, 1993.

[12] Gershon, N.D. and A. Nir, Effects ... through porous medium. Water Resources Research, 5, 830-839, 1969.

[13] Giddings, J.C., Kinetic origin of tailing in chromatography. Analytical Chemistry, 35, 1999-2002, 1963.

[14] Goltz, M.N. and P.V. Roberts, Interpreting ... non equilibrium models. Journal of Contaminant Hydrology, 1, 77-93, 1986.

[15] Green, R.E. et al., Solute ... adsorption. Proc. 2nd Sym. Fund. Trans. Phen. in Por. Med., IAHR-ISSS, Guelph, 2, 732-752, 1972.

[16] Gupta, R.K., R.J. Millington and A. Klute, Hydrodynamic ... dispersion. Jour.l of the Indian Soc. of Soil Sci., 21, 1-7, 1973.

[17] Hashimoto, I., K.B. Deshpande and H.C. Thomas, Peclet ... columns. Industrial Eng. Chemistry Fund., 3, 213-218, 1964.

[18] Horiuchi, M., Y. Inoue, N. Matsushita and T. Yoshida, The ... Layer. Proc. of Jap. Soc. of Civil Eng., 452, Vol. II-20, 1-10, 1992.

[19] Lapidus, L. and N,R, Amundson, Mathematics ... columns. Journal of Physical Chemistry, 56, 984-988, 1952.

[20] Lassey, K., Unidimensional ... analytical solutions. Water Resources Research, 3, 343-350, 1988.

[21] Matsubayashi, U., G. Velasquez and F. Takagi, Separation ... Relationships. Proc. of Hydr. Eng., JSCE, 35, 99-104, 1991.

[22] MacMahon, M.A. and G.W. Thomas, Chloride... cores. Soil Science Society American Proceedings, 38, 727-732, 1974.

[23] Macpherson, D.K. and A.J. Peck, Models of the effect ... catchment. Journal of Hydrology, 94, 163-179, 1987.

[24] Nielsen,D.R. and J.W. Biggar, Miscible ... information. Soil Science Society American Proceedings, 25, 1-5, 1961.

[25] Nkedi-Kizza, and others, Modeling ... oxisol. Water Resour.Research, 19, 691-700, 1983.

[26] Remson, I, G.M. Hornberger and F.J. Moltz, Numerical Methods in Sub-surface Hydrology. Wiley-Intersci., 6-121, 1971.

[27] Shimojima, E. and M.L. Sharma, On the transportation ... zone. Ann. Jap. Conf., JSCE, 48, 242-243, 1993. (In Japanese)

[28] Skopp, J. and A.W. Warrick, A two phase ... soils. Soil Science Society American Proceedings, 38, 545-550, 1974.

[29] Smiles, D. and J. Philip, Solute transport ... Implications. Soil Sci. Soc. of Am. Journal. 42, 537-544, 1978.

[30] Sugita, F. and R. Gillham, Effect ... transport. In Tracers in Hydrology, 171-178, Assoc. Rain. Sto. \& Infil. Tech., Tokyo, Jap., 1993.

[31] Taniguchi, M and M.L. Sharma, Solute and ... recharge rate. Journal of Hydrology, 119, 57-69, 1990.

[32] Toride, N., F. Leij and M. van Genuchten, Analytical ... zero-order production. Water Resour. Res., 29, 2167-2182, 1993.

[33] van Genuchten, M.Th. and P.J. Wierenga, Mass transfer ... solutions. Journal of Soil Science, 40, 473-480, 1976.

[34] van Genuchten, M.Th. and R.J. Wagenet, Two site/two ... solutions. Soil Sci. Society Am. Jour., 53, 1303-1310, 1989.

[35] Velasquez, G. T., On the Separation ... Contact Relationships. Masteral Thesis, Nagoya University, 1991.

[36] Velasquez, G. T., On the Role of ... Run-off Process Analyses. Doctoral Thesis, Nagoya University, 1994.

[37] Villermaux, J. and W.P.M. van Swaay, Modele ... stagnantes. Chem. Eng. Science, 24, 1097-1111, 1969. (In French)

[38] Zurmuhl, T., W. Durner and R. Herrmann, Transport ... columns, Journal of Contaminant Hydrology, 8, 111-133, 1991. 